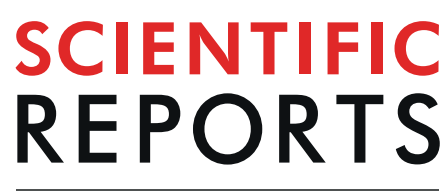

\title{
Surviving salt fluctuations: stress and recovery in Halobacterium salinarum, an extreme halophilic Archaeon
}

\begin{abstract}
P. Vauclare ${ }^{1,4}$, F. Natali ${ }^{2,3,4}$, J. P. Kleman ${ }^{1}$, G. Zaccai ${ }^{1,3}$ \& B. Franzetti ${ }^{1 *}$
Halophilic proteins subjected to below about $15 \%$ salt in vitro denature through misfolding, aggregation and/or precipitation. Halobacteria, however, have been detected in environments of fluctuating salinity such as coastal salterns and even around fresh water springs in the depths of the Dead Sea. In order to identify the underlying mechanisms of low salt survival, we explored the reactivation capacity of Halobacterium ( $\mathrm{Hbt}$ ) salinarum sub-populations after incubation in low salt media and recovery in physiological salt. Respiratory oxygen consumption was assessed in stressed cells and cell viability was estimated by Live/Dead staining and flow cytometry. In vivo neutron scattering experiments showed that the recovery of $\mathrm{Hbt}$ salinarum sub-populations exposed to severe low salt conditions is related to a rapid retrieval of functional molecular dynamics in the proteome. In the hypothesis that the observations on $\mathrm{Hbt}$ salinarum have wider relevance, they could be of key ecological significance for the dispersion of extremophiles when environmental fluctuations become severe.
\end{abstract}

Halophilic Archaea require hypersaline conditions (up to $5 \mathrm{M} \mathrm{NaCl}$ ) for optimal growth conditions found in various habitats on Earth, such as natural hypersaline lakes ${ }^{1}$, salterns or subterranean salt deposits ${ }^{2}$. Extreme halophilic Archaea like Halobacterium salinarum are of special interest because they resist the external osmotic pressure mainly by the accumulation of correspondingly high intracellular $\mathrm{KCl}$ concentrations ${ }^{3}$. Cellular accumulation of $\mathrm{K}^{+}, \mathrm{Cl}^{-}$, and exclusion of $\mathrm{Na}^{+}$requires energy dependent mechanisms achieved by $\mathrm{Na}^{+} / \mathrm{H}^{+}$antiporter systems and $\mathrm{K}^{+}$transport systems ${ }^{4}$. It was baffling to the scientific community that essential macromolecular interactions were not inhibited in the close to saturated salt cytosol of extreme halophiles and, furthermore, that salt concentration bellow $2 \mathrm{M}$ in which mesophilic microorganisms thrive resulted in halophilic enzyme inactivation ${ }^{5-7}$. A resolution to the puzzle was proposed from in vitro experiments on model enzymes, which revealed the requirement for high salt concentration to stabilize halophilic protein structures through solvation shells made up of hydrated salt ions ${ }^{8}$. The property is correlated with negatively charged protein surfaces, via an enrichment in acidic amino acids and marginal hydrophobic amino acids that favor repulsive interparticle forces to avoid aggregation in the high salt environment ${ }^{9,10}$. Site-directed mutagenesis of a halophilic protein from Haloferax volcanii and its mesophilic homologue and the subsequent NMR and thermodynamics characterizations indicated that surface aspartic and glutamic acids allow reducing the interaction surface between the protein and the solvent, which is beneficial in low water activity conditions to maintain an hydration shell ${ }^{11}$. Neutron scattering measurements of the molecular dynamic properties of the MalDH enzyme from Haloarcula. marismortui and of the native proteome from $\mathrm{Hbt}$. salinarum ${ }^{12-16}$ indicated that halophilic proteins display specific biophysical adaptation properties to high salt reflected by higher structural resilience, compared to mesophilic systems. It is likely, therefore, that most halophilic proteins display an obligatory requirement for high salt conditions in order to be active and stable. As a consequence, it is expected that intracellular ionic fluctuations in response to external concentration differences pose non-trivial problems for cellular biochemistry in extreme halophiles.

In coastal salterns extreme halophiles populations can be exposed to seasonal fluctuation in salt concentration, fresh water supply as well as transient rains and flooding episodes. Halophilic organisms exhibit a range of stress responses to counterbalance the deleterious effects induced by low salt. Hbt. salinarum accumulates a heat shock

${ }^{1}$ University Grenoble Alpes, CNRS, CEA, IBS, F-38000, Grenoble, France. ${ }^{2}$ CNR-IOM, OGG, F-38042, Grenoble, France. ${ }^{3}$ Institut Laue Langevin (ILL), F-38042, Grenoble, France. ${ }^{4}$ These authors contributed equally: P. Vauclare and F. Natali. *email: franzetti@ibs.fr 
protein in the cytosol (the thermosome, known to stabilize proteins during thermal stress) as well as low salt stress specific chaperonin complex called Ssp $45^{17}$. Proteasome complexes, involved in the clearing of misfolded or damaged proteins are also induced by moderate hyposaline stress $(\sim 2.5 \mathrm{M} \mathrm{NaCl})^{18}$. Other responses include down-regulation of the translational apparatus and modulation of the expression of genes encoding for enzymes associated with the primary metabolism ${ }^{19,20}$. Furthermore, the expression of many genes encoding for peptides and ion transporters, such as those that regulate $\mathrm{K}^{+}$and $\mathrm{Na}^{+}$concentration in the cytosol, are salt- dependent ${ }^{21,22}$.

In vitro, halophilic proteins subjected to low salt denature through misfolding, aggregation and/or precipitation. In its natural environment, however, Hbt salinarum is exposed to large salt fluctuations. In this context, we explored the reactivation capacity of $\mathrm{Hs}$ sub-populations after incubation in low salt media, $2.5 \mathrm{M}$ down to $0.5 \mathrm{M}$ $\mathrm{NaCl}$, and recovery in physiological salt $(4.2 \mathrm{M} \mathrm{NaCl})$. Respiratory oxygen consumption was assessed in stressed cells and cell viability was estimated by Live/Dead staining and flow cytometry. The molecular dynamics of the proteome represents a good indicator of protein functionality. In vivo neutron scattering experiments showed that the recovery of Hbt salinarum sub-populations exposed to severe low salt conditions is related to a rapid retrieval of functional molecular dynamics. The observations are of key ecological relevance in the context of climate change and the dispersion of extremophiles in fluctuating environments.

Also, Dawson et al..$^{23}$ observed that halophilic archaea regulate the proportion of unsaturated diacylglycerol glycerol diethers (DGDs) in the membrane lipids according to salt fluctuations in the environment.

Compatible solutes may also exist in extreme halophilic Archaea as suggested by comparative genomic analyses ${ }^{24}$ but these organisms display a limited capacity to adapt to salt concentration below their optimum ${ }^{4}$. In the case of Hbt. salinarum we have shown that below $2 \mathrm{M} \mathrm{NaCl}$ (a value which induces unfolding of the model Haloarcula marismortui enzyme, MalDH, in absence of cofactors) halophilic cells suffer a high mortality rate ${ }^{15}$. In the same study, molecular dynamics parameters of the proteome were measured in vivo by neutron scattering for cultures exposed to various lower salt concentrations. Already at $2.5 \mathrm{M}$ salt, significant protein molecular dynamics perturbations were observed, similar to those in heat stressed Hbt. salinarum cells ${ }^{14}$. These observations suggested that alteration in cytosolic protein solubility/stability represent a major cause of cellular stress in low salt conditions. It is assumed that such modification would cause irreversible damage, thus preventing cellular reactivation when optimal environmental hypersalinity is restored. Considering the stress effect of low salt condition on halophilic systems, it is surprising that several studies highlighted the presence of halophilic Archaea in low salt conditions in soils, human or plant microbiomes and in the stratosphere ${ }^{25-29}$. In this work, we explored the reactivation capacity of Hbt. salinarum sub-populations after incubation in low salt conditions. We studied different cell properties after salt shock $(2.5 \mathrm{M}$ down to $0.5 \mathrm{M} \mathrm{NaCl})$ and recovery in high salt $(4.2 \mathrm{M} \mathrm{NaCl})$. Cell viability was estimated by Live/Dead staining and flow cytometry, which allowed us to follow in real time multiple cellular parameters including cell membrane integrity. Because Hbt. salinarum is an aerobic heterotroph, we also assessed the respiratory oxygen consumption of stressed cells. Hbt. salinarum proteome molecular dynamics under different conditions was then measured in vivo by neutron scattering. In previous work, effects of the environment on protein dynamics and its direct relevance for biological activity ${ }^{30}$ was illustrated by neutron scattering studies of proteome molecular dynamics, in vivo, on bacterial cells adapted to different physiological temperatures ${ }^{31}$. In the study, the mean structural resilience of the proteome, expressed as an effective force constant, was found to be adapted in order to maintain flexibility appropriate for activity at the physiological temperature. Thus, psychrophiles had 'softer' structures, flexible at low temperatures, while the thermophile proteome was 'harder' to maintain stability at high temperatures. The measure of flexibility converged to a constant value at physiological temperature for all the organisms examined. Similarly, the current study established that molecular dynamics in Hbt. salinarum is adapted to physiological high salt and constitutes a sensitive measure of cellular distress at the molecular level when the salt concentration drops. Here, the neutron scattering study revealed that, in the cytosol of reactivable cells, the necessary condition of functional molecular dynamics was rapidly restored after being significantly affected by low salt stress. With this approach, we obtained evidence that sub-populations of $\mathrm{Hbt}$. salinarum can survive severe low salt conditions to start multiplying again when the salt concentration rises back to physiological values. These observations may have general ecological significance in the context of dispersion of extremophiles in fluctuating environments.

\section{Results}

Hbt. salinarum cell growth during low salt stress and re-inoculation in high salt optimal condi-

tions. In extreme halophiles, low salt conditions elicits the induction of stress response systems suggesting that part of the halophilic population could tolerate significant diminutions of environmental salt conditions. To test this hypothesis, Hbt. salinarum cultures were diluted in growth media containing different $\mathrm{NaCl}$ concentrations and growth was measured for 7 days. Cells exposed to $2.5 \mathrm{M} \mathrm{NaCl}$ maintained their ability to divide for a few days (Fig. 1). The growth rates, however, were up to 5 to 8 times lower than for control cells in $4.2 \mathrm{M} \mathrm{NaCl}$. Growth then slowed down progressively, suggesting that cells entered a phase similar to a stationary phase. The stressful effect of low salt concentration appeared to be particularly pronounced at concentrations lower than $1.5 \mathrm{M} \mathrm{NaCl}$ (Fig. 1A). Below 1.5 M, growth curves exhibit a typical logarithmic biphasic killing pattern described in many bacteria exposed to harmful environmental stress ${ }^{32}$, which may reflect an initial exponential phase corresponding to a sensitive population and a second phase with a much slower decline curve follow by a plateau. Interestingly, we observed that all stressed cells inoculated into hypersaline physiological medium switch back to a normal growing cell population after a given time period (Fig. 1B), suggesting that recovered cells, including those that have undergone extreme low-salt shock, possess the capacity to multiply again. Cells incubated in $2.5 \mathrm{M}$ started to grow instantaneously. A lag phase of three days was recorded for cells incubated during 1 hour in $0.5 \mathrm{M} \mathrm{NaCl}$ (Fig. 1B1) and of 7 days for cells incubated during one week in $1.5 \mathrm{M}, 1 \mathrm{M}$ or $0.5 \mathrm{M} \mathrm{NaCl}$ (Fig. 1B2). The observations suggest that part of the low salt stressed cells can undergo a reactivation process when they encounter optimal saline conditions. 

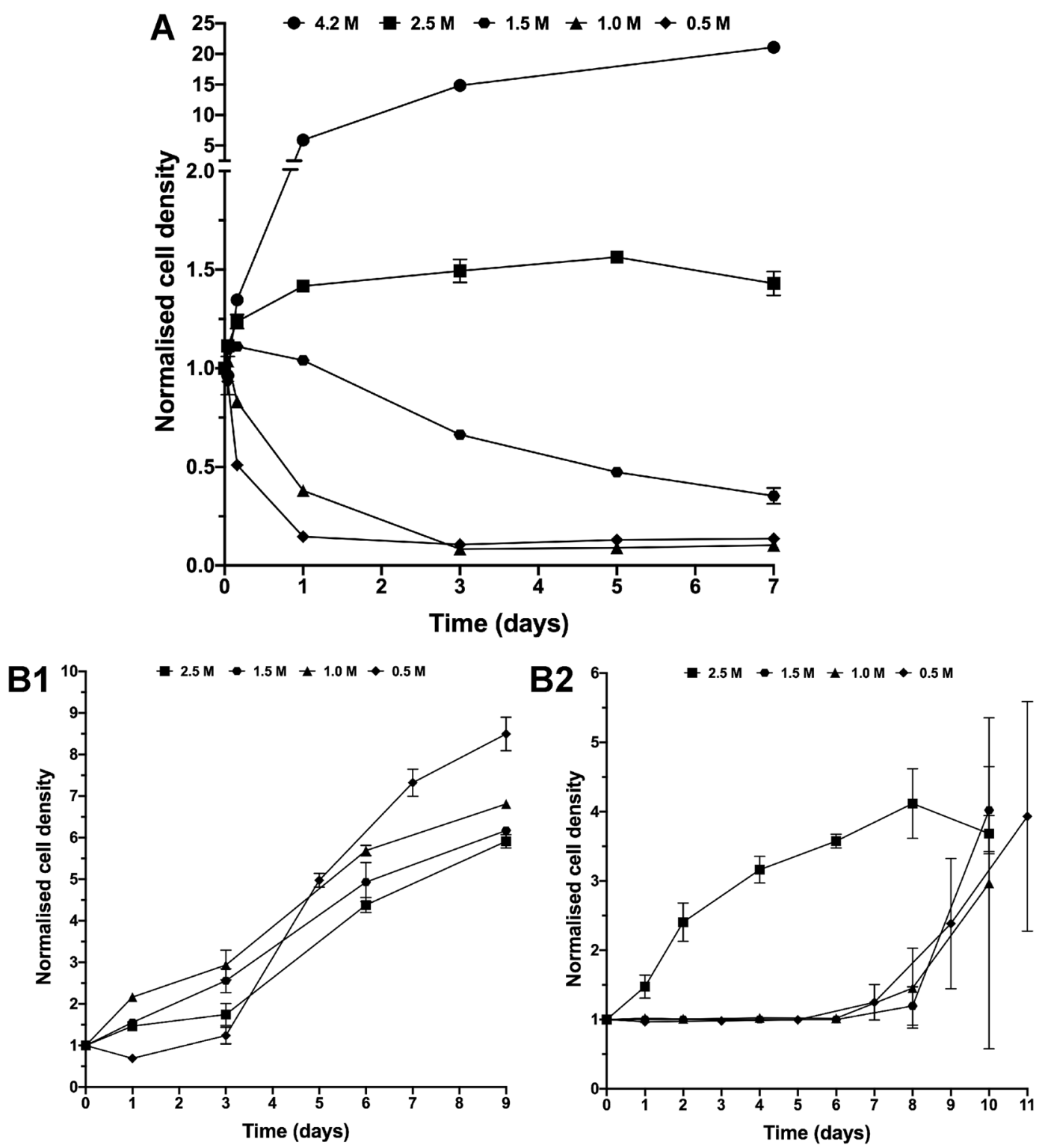

Figure 1. (A) Normalized cell density of Halobacterium salinarum as a function of time for different salt $(\mathrm{NaCl})$ concentrations. Cells grown in $4.2 \mathrm{M} \mathrm{NaCl}$ were harvested then cultivated at $37^{\circ} \mathrm{C}$ under aerobic conditions in rich medium with $2.5 \mathrm{M}, 1.5 \mathrm{M}, 1.0 \mathrm{M}$ and $0.5 \mathrm{M} \mathrm{NaCl}$. (B) Growth kinetics of Hbt. salinarum during recovery. Cells were shocked in $2.5 \mathrm{M}(\boldsymbol{\square}), 1.5 \mathrm{M}(\mathbf{\Delta}), 1.0 \mathrm{M}(\bullet)$ and $0.5 \mathrm{M}(\mathrm{x}) \mathrm{NaCl}$ for 1 hour (B1) or 7 days (B2) and then were incubated in $4.2 \mathrm{M} \mathrm{NaCl}$ for recovery. Growth was monitored as optical density of the culture. Each experiment was carried out in triplicate. Errors bars represent standard errors.

Flow cytometry analysis of $\mathrm{Hbt}$. salinarum cells structural integrity under low salt stress and high salt reactivation. The viability of stressed $\mathrm{Hbt}$. salinarum cells was assessed by flow cytometry analysis ${ }^{33}$. Mainly used for eukaryote and bacterial analysis, the extreme physico-chemical conditions around $\mathrm{Hbt}$. salinarum were limiting for the application of flow cytometry. The method was tested for the first time on extreme halophiles in this study and we confirmed its effectiveness by using SYTO 9/Propidium iodide staining, a powerful indicator of membrane integrity (LIVE/DEAD ${ }^{\mathrm{TM}}$ Baclight $^{\mathrm{TM}}$ bacterial viability kit, Molecular Probes) ${ }^{14}$. Propidium iodide (PI) fluorescent nucleic acid stain identifies damaged cells that lost membrane integrity (PI+) (especially significant considering the key role of the membrane in bioenergetics and maintenance of the electrochemical potential of $\mathrm{K}^{+}, \mathrm{Na}^{+}$, and $\mathrm{H}^{+}$ions ${ }^{33}$ ), while SYTO9 DNA labeling distinguishes cells from debris, empty cell ghosts, and DNA-damaged subpopulations (Syto-).

Aspects of different cell populations are shown in scanning and transmission electron micrographs in (Fig. S1). The cell populations were analyzed after 1 hour (Fig. 2A) and 24 hours (Fig. 2B) incubation at $37^{\circ} \mathrm{C}$ under agitation in growth media containing 4.2 (control), 2.5, $1.5,1.0$ and $0.5 \mathrm{M} \mathrm{NaCl}$, respectively. As expected, the accumulation of PI+/PI-, Syto - and lytic cells increased proportionally to the diminution of environmental salt concentration and stress duration. After moderate salt stress $(2.5 \mathrm{M})$, however, a significant part of the initial 

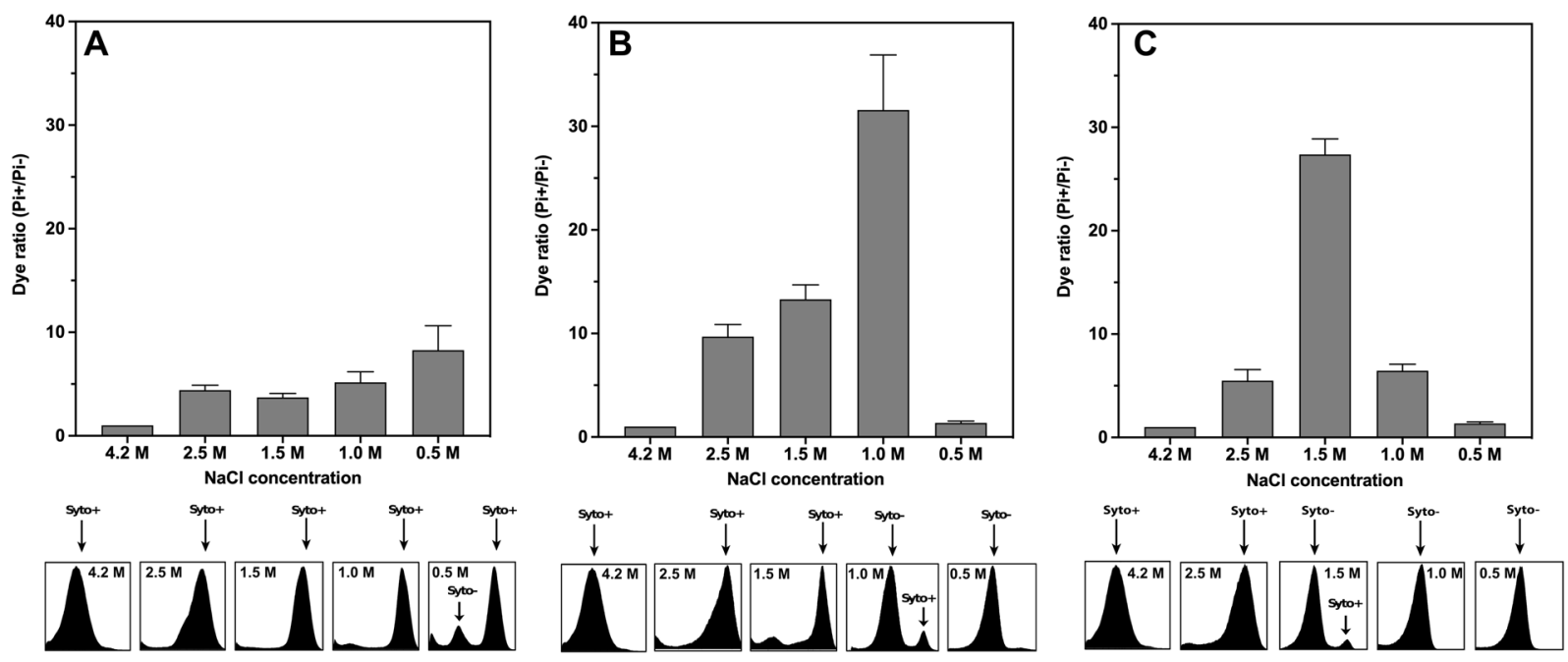

Figure 2. Flow cytometry analysis of membrane integrity (PI labeling) and DNA staining (Syto labeling) of Hbt. salinarum cells. The cells were incubated for 1 hour (A) and 24 hours (B) and 7 days (C) in standard growth medium $(4.2 \mathrm{M} \mathrm{NaCl}$, control) or in low $\mathrm{NaCl}$ conditions $(2.5 \mathrm{M}, 1.5 \mathrm{M}, 1.0 \mathrm{M}$ and $0.5 \mathrm{M}$ At the top, $\mathrm{PI}+$ (membrane integrity) cell populations detected at $645 \mathrm{~nm}$ (PI). At the bottom, histograms of Syto9 (DNA labelling) staining showing changes in the fluorescence intensity (at $528 \mathrm{~nm}$ ) of cells exposed to various salt concentrations. Damaged (Syto-) and undamaged DNA (Syto+) populations are indicated by arrows. Each experiment was carried out in triplicate. Errors bars represent standard errors.

population remained intact, even after 7 days (Fig. 2C). It is noteworthy that after 1 hour in $0.5 \mathrm{M} \mathrm{NaCl}$ a significant proportion of the cells retained structural and DNA integrity (Fig. 2A). After a prolonged incubation in low salt concentrations ( 24 hours and 7 days), the majority of the harvested cells displayed considerable DNA damage and membrane injury (Fig. 2B,C). However, for cells incubated in $\mathrm{NaCl} 0.5 \mathrm{M}$ for 24 hours or in $\mathrm{NaCl} 1 \mathrm{M}$ and $0.5 \mathrm{M}$ for 7 days, the $(\mathrm{Pi}+/ \mathrm{Pi}-)$ decreased significantly to reach values close to that of unstressed cells. A possible explanation for these results is that most cells are lysed under these extreme conditions, and the signal is dominated by a small fraction of surviving cells.

To monitor reactivation, low salt $(0.5 \mathrm{M} \mathrm{NaCl})$ stressed $\mathrm{Hbt}$. salinarum samples were subsequently analyzed after harvesting and incubation for 1 and 24 hours, respectively, back in physiological saline medium (Fig. 3). After 1 hour incubation $(\mathrm{CR}-1 \mathrm{~h})$, the $(\mathrm{Pi}+/ \mathrm{Pi}-)$ ratio decreased indicating less membrane damage compared to the initial stressed cells; on the other hand, the Syto- and Syto + pattern while similar to that of the stressed cells (Fig. 2A) shows a gradual shift towards Syto+ values indicating fewer cells with DNA damage. The tendency is confirmed in CR-24h, in which only one population is observed with undamaged DNA and intact membranes. Taken together, these results indicated that a significant sub-population of Hbt. salinarum cells can maintain its membrane integrity and is competent to recover from low salt stress.

Time-lapse microscopy of low salt stressed $\mathrm{Hbt}$ salinarum cell populations during high salt reactivation. In an exploration of Hbt. salinarum reactivation, we examined the evolution of cell morphology by time-lapse microscopy (Dichroic Interference Contrast imaging; DIC) during recovery in $4.2 \mathrm{M} \mathrm{NaCl}$ following incubation in $0.5 \mathrm{M} \mathrm{NaCl}$ (Fig. 4) and $2.0 \mathrm{M} \mathrm{NaCl}$ (Fig. S2) growth medium for 1 hour. Stressed cells $(0.5 \mathrm{M} \mathrm{NaCl})$ exhibited a round shape about $1 \mu \mathrm{m}$ in diameter during the first 16 hours of culture in hypersaline conditions. At about 32-36 hours small islets of cells regaining the typical rod-like shape of Hbt. salinarum asynchronously appeared. Interestingly, rod-shaped cell formation did not follow classical binary fission, used by most Archaea for cell division. Cell division rather initiated from flat sacculi that eventually acquired conventional rod morphology. The rod-shape cells then elongated and split into two daughter cells. The cycle was repeated as long as cells were cultured under favorable conditions, consistent with growth rates presented in Fig. 1.

Assessment of the metabolic state of stressed and reactivated $\boldsymbol{H}$. salinarum populations. The effect of low salt exposure on respiration is shown in Fig. 5 (light grey bars). We observed that the respiratory sensitivity of Haloarchaea is $\mathrm{NaCl}$ concentration and time-dependent. As illustrated in Fig. 5, lower salt concentration led to a loss of respiratory rate; a phenomenon amplified when the incubation time increased. The longer the shock, the higher the minimum value of salt concentration for which respiration rate reached zero. The $\mathrm{O}_{2}$-uptake rate of stressed cells decreased notably for cells exposed to below $1.5 \mathrm{M} \mathrm{NaCl}$ for 24 hours or 7 days. The progressive loss of respiration, under these conditions, parallels slow or stopped Hbt. salinarum growth in the same conditions (Fig. 1). Figure 5 also shows the respiration activity of low salt stressed Hbt. salinarum cells incubated back in a high salt medium (dark grey bars). It shows that the respiratory activity increases rapidly after transfer to $4.2 \mathrm{M} \mathrm{NaCl}$ physiological medium (Fig. 5, RRA), even for cells exposed for a long time to extreme low salt concentration (Fig. 5C,D). In all cases, respiration increases instantaneously including for the longest shock (7 days) at the lowest salt concentration $(0.5 \mathrm{M} \mathrm{NaCl})$. This cannot be the result of undesired contaminations, as demonstrated by control measurements of the recovery medium alone (data not shown). The observations 




Figure 3. Flow cytometry analysis of membrane integrity (PI labeling) and DNA staining (Syto labeling) of $\mathrm{Hbt}$. salinarum recovered cells. The cells were incubated in $4.2 \mathrm{M} \mathrm{NaCl}$ (control) or after low salt shock $(0.5$ $\mathrm{M}-1 \mathrm{~h}$ ) for one hour followed by cell recovery in $4.2 \mathrm{M} \mathrm{NaCl}$ for 1 hour (CR-1h) and 24 hours (CR-24h). At the top, PI+ (membrane integrity) cell populations detected at $645 \mathrm{~nm}$ (PI). At the bottom, histograms of Syto 9 (DNA labelling) staining showing changes in the fluorescence intensity (at $528 \mathrm{~nm}$ ) of cells exposed to various salt concentrations. Damaged (Syto-) and undamaged DNA (Syto+) populations are indicated by arrows. Each experiment was carried out in triplicate. Errors bars represent standard errors.

suggested that a significant part of the cell population is still able to produce energy even during a brutal salt shock or when exposed to prolonged periods of stress at moderately low salt concentrations.

In vivo molecular dynamics parameters in low salt stressed and high salt reactivated samples. As discussed previously, the molecular dynamic state of the cellular proteome represents a robust indicator of cellular fitness ${ }^{14,15,31}$. Macromolecular denaturation through unfolding leads to lower effective force constants indicating less rigid structures. According to in vitro experiments on a model halophilic protein and to our previous work on $\mathrm{Hbt}$. salinarum cells exposed to moderate salt stress $(2 \mathrm{M} \mathrm{NaCl})^{15}$, a drop in intracellular $\mathrm{K}$ concentration should induce important damage to the proteome. Here, we performed in vivo neutron scattering experiments to characterize the molecular dynamics parameters of the Hbt. salinarum proteome exposed to low salt.

Samples were measured on the IN13 spectrometer at the ILL ( $8 \mu \mathrm{eV}$ energy resolution sampling a $0.1 \mathrm{~ns}$ time scale), as described in Vauclare et al. ${ }^{15}$. Sample preparation for the neutron experiments is described in Materials and Methods. A centrifugation step prior to sample load in the measurement cell eliminated most of cell debris and lysed material. We considered, therefore, that the measured signal was dominated by the fraction of intact cells in the low salt stress samples to provide relevant information on their functional state. The experiments displayed in Fig. 6 correspond to Hbt. salinarum cultures exposed to $2.5 \mathrm{M}$ and $0.5 \mathrm{M} \mathrm{NaCl}$ concentrations for 1 hour or 24 hours. The effective average force constant $\left\langle\mathrm{k}^{\prime}\right\rangle(\mathrm{N} / \mathrm{m})$ stabilizing macromolecular structures within the cell was calculated from the temperature dependence of mean square displacements (MSD). The $<k^{\prime}>$ value fell progressively with lower salt concentrations and longer exposure times, indicating a softening of the resilience of macromolecular structures, as observed previously for heat-stressed cells and cells exposed to a moderate low salt stress for $1 \mathrm{~h}^{14,15}$. The scattering vector modulus (Q) range of IN13 extends from $0.3 \AA^{-1}$ to almost $5 \AA^{-1}$, corresponding to a length scale from a few ångströms (at low Q) to a fraction of an ångström. The analysis was limited to the low Q end, where the time-length window is appropriate for MSD of macromolecular internal motions as well as the larger fluctuation amplitudes involved in unfolding processes (Fig. S3). Unfolded proteins have been measured by neutron scattering to display lower resilience than folded states ${ }^{34}$ so that the effective force constants in the low salt samples in Fig. 6 indicated less rigid macromolecular structures, reflecting perturbation in the folding state of a significant part of the Hbt. salinarum proteome. Even if the unfolded structures tended to aggregate, it is not unlikely on the time scale of internal motions that such aggregates would be less resilient than the compact cores of native states ${ }^{34}$. We subsequently measured the effective average force constants of $\mathrm{Hbt}$. salinarum after 1 hour and 24 hours of high salt recovery (Fig. 7). The $2.5 \mathrm{M} \mathrm{NaCl}$ and $0.5 \mathrm{M} \mathrm{NaCl}$ concentrations, with further incubation at $4.2 \mathrm{M} \mathrm{NaCl}$ for 1 hour, were chosen to represent a moderate and an extreme stress conditions. The $\left\langle\mathrm{k}^{\prime}\right\rangle$ values for the low salt stressed samples are given by the dashed blocks (data from Fig. 6). The $\left\langle\mathrm{k}^{\prime}\right\rangle$ values of the recovered samples (solid grey blocks) have themselves recovered the physiological value of 


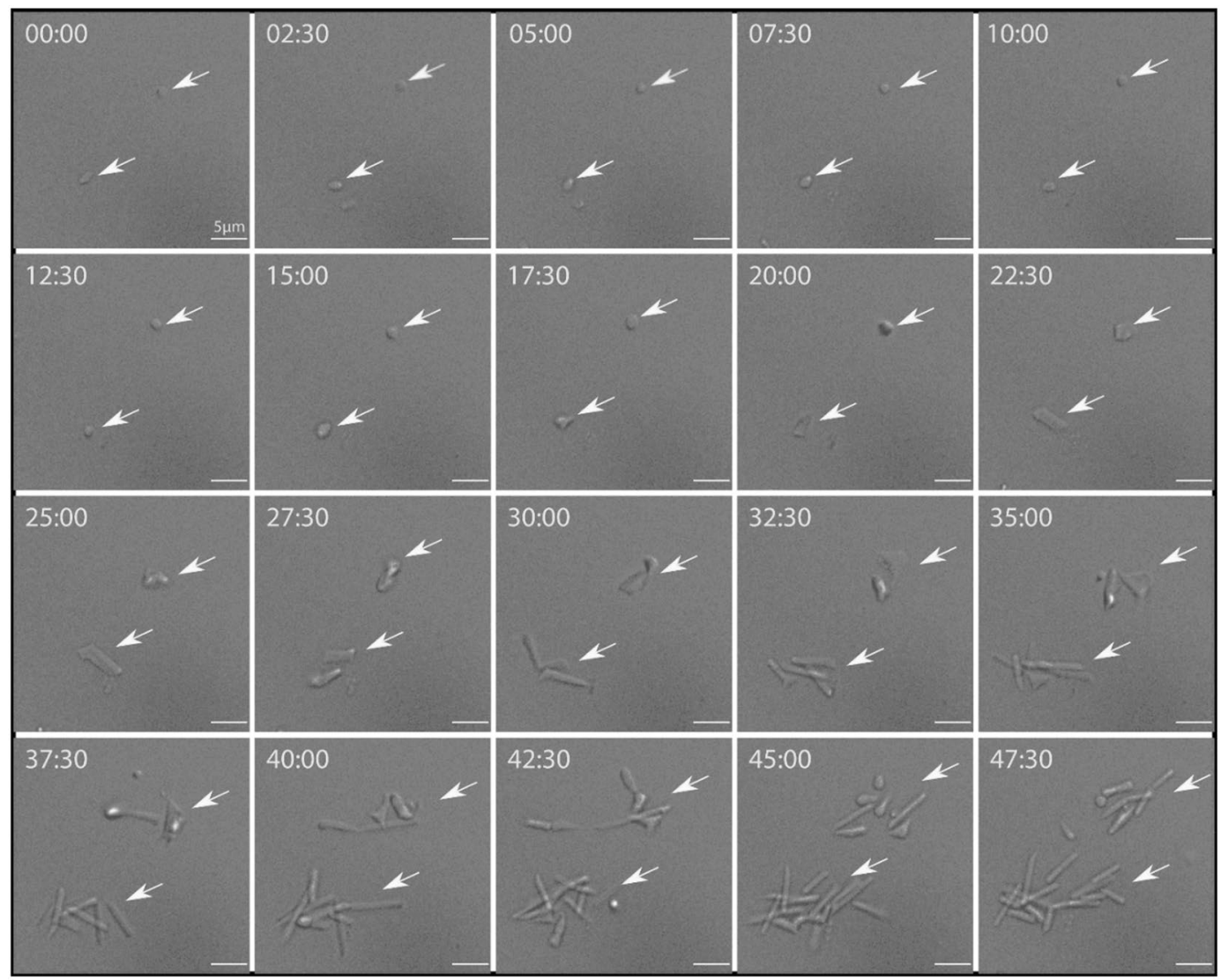

Figure 4. Time-lapse light microscopy. Hbt. salinarum morphological changes were monitored during recovery in $4.2 \mathrm{M} \mathrm{NaCl}$ growth medium after a low-salt shock $(1 \mathrm{~h})$ at $0.5 \mathrm{M}$. Cells were grown at $37^{\circ} \mathrm{C}$ in the microscopy chamber and observed by Dichroic Interference Contrast. Images were collected every 30 minutes over a period of 48 hours.

the $4.2 \mathrm{M} \mathrm{NaCl}$ control (white block), indicating that a major part of the cells that resisted the low salt conditions had rapidly regained a favorable internal macromolecular environment, a necessary precondition for the recovery of membrane integrity, respiration and viability.

\section{Discussion}

Low salt concentrations, in vitro, were found to be strongly destabilizing for model halophilic proteins ${ }^{8,10}$. Previous neutron scattering experiments established this was also the case in the cytosol of Hbt. salinarum exposed to moderate low salt ${ }^{15}$. In this work, we extended the neutron scattering observations to extreme low salt shock. And, as expected, a more substantial dynamic perturbation of the proteome was observed. Furthermore, we characterized cellular and physiological modifications resulting from low salt stress and shock. Hbt. salinarum exposed to low salt stops growing. Most cells adopt a spherical shape; an increasing fraction of dead cells and cells with damaged membranes is detected together with a dramatic decrease in residual respiratory activity. Strikingly, even for the sample exposed to $0.5 \mathrm{M} \mathrm{NaCl}$, we observed the return of respiration and proteome molecular dynamics was rapidly restored to control values already after incubation for 1 hour back in $4.2 \mathrm{M} \mathrm{NaCl}$. The evolution of cell architecture suggested that cell division property is recovered after several hours incubation in 4.2 M NaCl. Round-shaped cells with a dense cytosol progressed to undefined flat saccules; after about 32 to 36 hours, small islands of rod-shaped cells, typical of Hbt. salinarum, appeared asynchronously and continued to divide. This cycle was repeated, consistent with the growth rate measurements. The observations indicated that despite the harsh low salt shock exposure, which significantly affected the molecular dynamics of the proteome and consequently cell metabolism, a fraction of cells maintained their capacity to recover. Different non-exclusive hypotheses could explain the observation: (1) large intracellular molecular assemblies, like chaperonins, proteasomes, peptidases, that were shown to be more resistant to low salt conditions in vitro ${ }^{17,18,35}$ trigger the recovery; (2) low salt conditions induce the accumulation of protective stress response molecules, such as chaperones or compatible solutes; (3) The formation of persisters, dormant stress-tolerant phenotypic variants first identified while investigating antibiotic resistance in bacteria ${ }^{36}$. Even though the latter hypothesis is supported by the biphasic time-kill curves, further investigation will be required to establish whether or not $H$. salinarum low salt stress survivors share other properties specific to persisters ${ }^{37}$. 

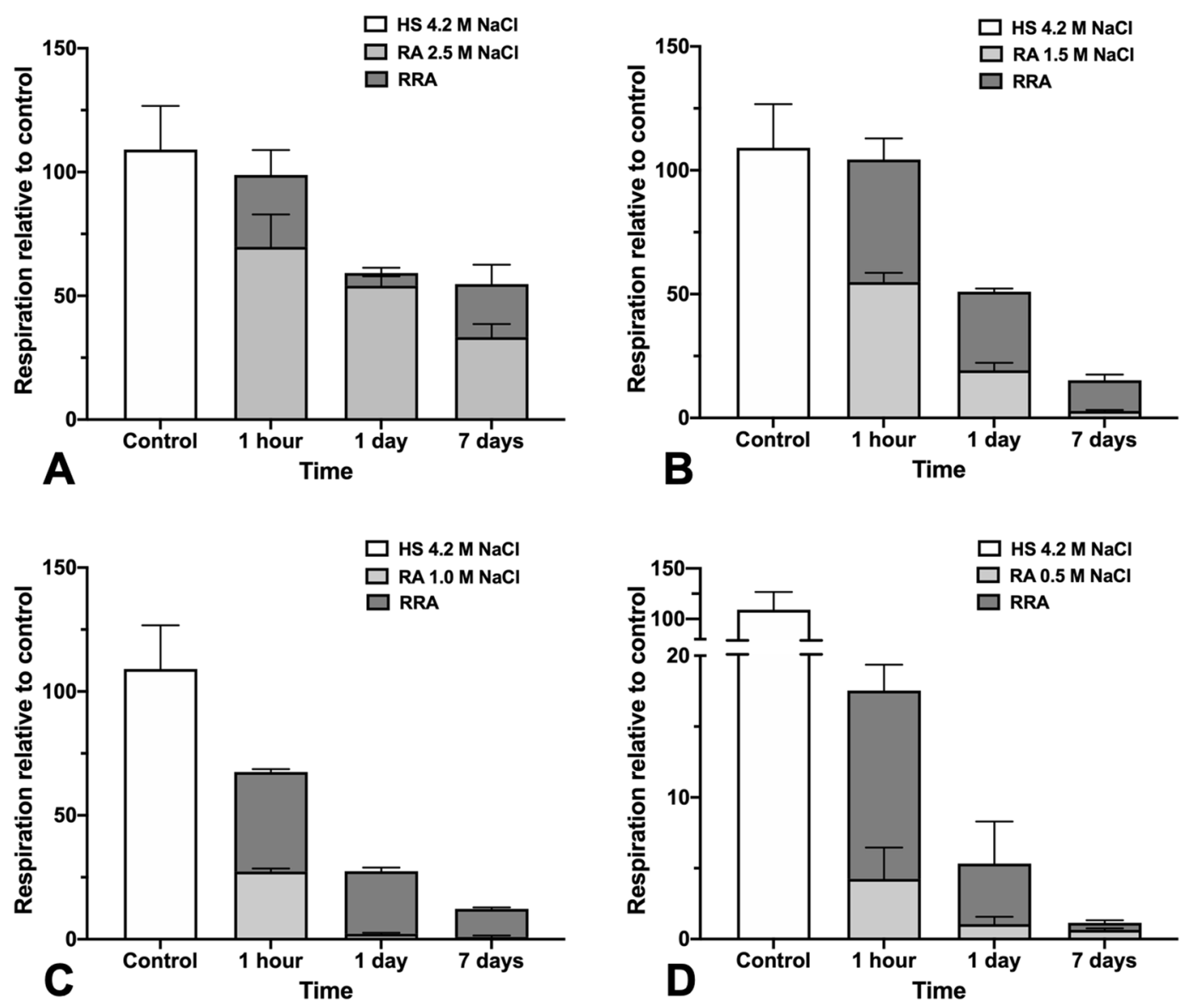

Figure 5. Respiration rate of Hbt. salinarum. Respiration activity (RA, light grey) of cells in low-salt shock $(2.5 \mathrm{M}, 1.5 \mathrm{M}, 1 \mathrm{M}$ and $0.5 \mathrm{M} \mathrm{NaCl})$ measured at 1 hour, 24 hours and 7 days and recovery respiration activity (RRA, dark grey) immediately after transfer of stressed cells in standard $4.2 \mathrm{M} \mathrm{NaCl}$ medium. Oxygen consumption of cells was determined at $37^{\circ} \mathrm{C}$ using a Unisense oxygen sensor. The experiment starts with basal respiration of unstressed cells (HS 4.2). Harvested cells were then exposed to various low-salt concentrations ((A) $2.5 \mathrm{M} \mathrm{NaCl}$, (B) $1.5 \mathrm{M} \mathrm{NaCl}$, (C) $1.0 \mathrm{M} \mathrm{NaCl}$ and (D) $0.5 \mathrm{M} \mathrm{NaCl}$ ) during different periods of time for respiration measurement. Finally, oxygen consumption of recovered cells was measured immediately after incubation of stressed cells in $4.2 \mathrm{M} \mathrm{NaCl}$. Basal respiration of unstressed cells was set to $100 \%$ in each case. Results are mean values for three independent experiments. Time on the $\mathrm{x}$-axis corresponds to the low-salt incubation time. Note that different scales were used in figure (D).

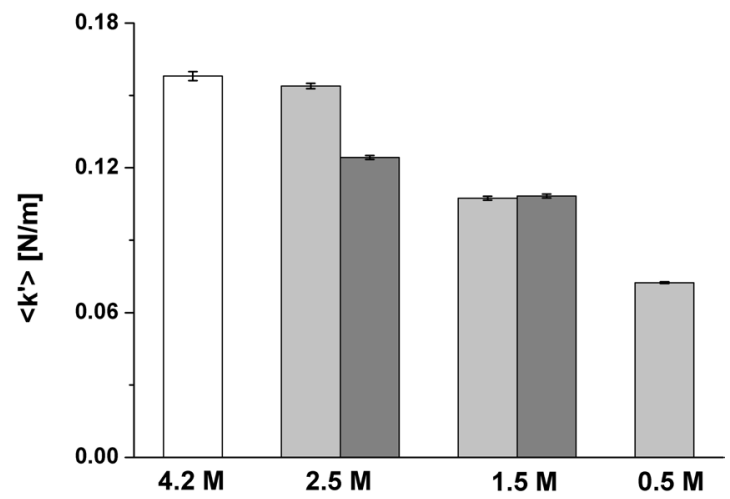

Figure 6. Mean effective force constant $\left\langle\mathrm{k}^{\prime}\right\rangle$ of $\mathrm{Hbt}$. salinarum, for different conditions. Columns represent values for a given salinity and incubation time ( 1 hour, light grey or 24 hours, dark grey). The white column is the control sample at physiological salt molarity. Errors bars represent standard errors. 


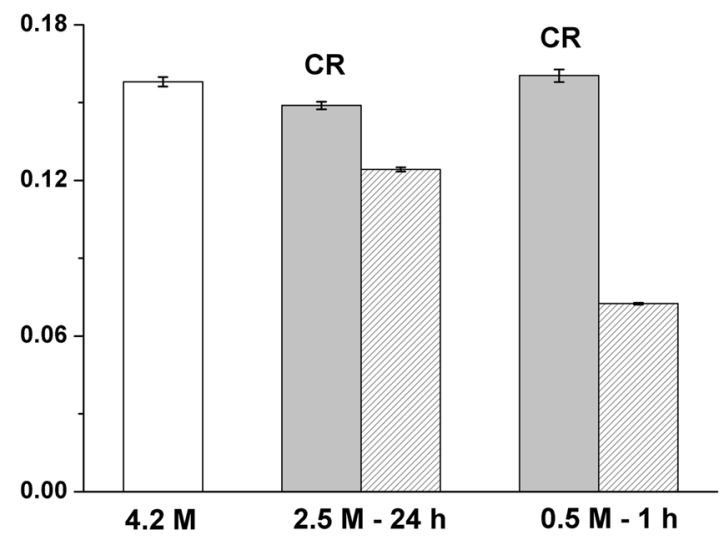

Figure 7. Mean effective force constant $<\mathrm{k}^{\prime}>$ of Hbt. salinarum. The reactivated samples (CR, solid grey blocks) are compared to the corresponding value under stress (dashed patterns) and the control (white column). Errors bars represent standard errors.

The neutron scattering results showed that the presence of cells that could be reactivated in low salt stress populations $(2.5 \mathrm{M}$ and $0.5 \mathrm{M} \mathrm{NaCl})$ was not due to functional molecular dynamics parameters being maintained during the stress event. This implies that a modified molecular dynamics landscape, reduced metabolic activity and morphological changes (in the case of the $2.5 \mathrm{M}$ sample) did not prevent the recovery of Hbt. salinarum cells from reactivating even if the perturbation were maintained for long periods, and that the reactivation process correlated with the restoration of functional molecular dynamic parameters. The phenomenon can be rapid in the case of short stress, consistent with the salt-dependent halophilic protein solvation/activation hypothesis ${ }^{7}$. The rapid recovery, after incubation in physiological salt, of proteome molecular dynamics and respiration indicates that the perturbation is reversible. Recovery could be due to the restoration of the favorable halophilic protein-solvent interactions at high salt and/or to de novo synthesis of key enzymes by the translation machinery, both processes fueled by intact membrane ion pumps and respiratory complexes. Note that other cellular functions may be much slower to appear as suggested by the later onset of cell division.

In this work, we highlighted the capacity of specific Hbt. salinarum cells to overcome the physico-chemical damage of low salt on halophilic protein molecular dynamics in order to recover when put back in physiological medium. The observation contributes to the understanding of how halophilic Archaea, exposed to important salt fluctuations occurring in their natural environments, can survive and disseminate in low salt conditions, such as in soils, human or plant microbiomes and in the stratosphere, where they have been shown to occur ${ }^{25-29}$. The characterization of in cellular molecular dynamics of stressed and reactivated cells illustrated the physico-chemical aspects of the survival process. The determination of the cell biology mechanisms involved in the selection and reactivation of resistant cells is a major challenge to be tackled in the future, in particular by combining single cell metabolomics and proteomics.

\section{Materials and Methods}

Hbt. salinarum cells: cultivation and growth conditions. Halobacterium salinarum R1 (DSM 671) is the hyperhalophilic archaea strain used for the study. Cells were grown at $37^{\circ} \mathrm{C}$, with shaking at $110 \mathrm{rpm}$ in standard hypersaline medium containing $4.2 \mathrm{M} \mathrm{NaCl}, 160 \mathrm{mM} \mathrm{MgSO}_{4}, 10 \mathrm{mM}$ Trisodium citrate and $26 \mathrm{mM} \mathrm{KCl}$ (Oesterhelt and Stoeckenius 1974) under aerobic conditions. For salt stress shock experiment, cells were grown to the mid exponential phase (OD $600 \sim 0.5-0.8)$ then centrifuged at $\sim 4000 \times$ g. For low salt stress experiments, the cell pellets were suspended in standard cultivation medium containing the desired $\mathrm{NaCl}$ concentration and cells were further cultivated for one hour, 24 hours and 7 days at $37^{\circ} \mathrm{C}$ under agitation. For the reactivation process, stressed cells were pelleted again and re-suspended in standard hypersaline media and cultivated at $37^{\circ} \mathrm{C}$ with shaking. The impact of the remaining salt concentration in the cellular paste on the final $\mathrm{NaCl}$ concentration of the medium used to stress the cells is negligible, as the pellet was resuspended at least in 1000 volumes of low-salt buffer.

SYT09/propidium iodide assay by flow cytometry analysis. Flow cytometry was applied to follow multiple cellular parameters such as cell membrane integrity, in real time during low salt and recovery at high salt. Cells were centrifuged and the pellets were suspended in washing buffer $(50 \mathrm{mM}$ Tris- $\mathrm{HCl} \mathrm{pH} 7.5 ; 70 \mathrm{mM}$ $\mathrm{KCl}$ and $80 \mathrm{mM} \mathrm{MgSO}_{4}$ ) with a similar salt concentration to the one used during the cultivation (control or salt stress). The operation was done in triplicate for each point. Samples were stained with live stain (SYTO 9) during 15 minutes at $37^{\circ} \mathrm{C}$ and the dead stain (propidium iodide: PI) was added just before analysis. Cells with damaged membranes will be penetrated by both stain SYTO 9 and PI. Flow cytometric analyses were carried out using the MACSQuant VYB (Miltenyi Biotec SAS, Bergisch Gladbach, Germany) equipped with three lasers, two scatter (FSC, SSC) and up to eight fluorescent channels. Green (SYTO 9) and red (PI) fluorescence, from cells excited by a $488 \mathrm{~nm}$ laser, was collected through a 525/50-nm or a $655-730-\mathrm{nm}$ bandpass filter, respectively. A MACSQuantify software was used to assess the mortality rate in the different physiological conditions as described ${ }^{15}$. Manual gating of flow cytometry data was carried out on the basis of Pi and syto9 fluorescence intensity to discriminate $\mathrm{PI}+/$ Syto + population from $\mathrm{PI}-/$ Syto - populations. Flow cytometry histogram profiles allow to differentiate Syto- population (low fluorescence yield) which are at least partly depleted in their DNA 
content from normal DNA content population (Syto+). Damaged or dead cells were determined from the ratio of PI+ (damaged membrane)/PI- (intact membrane).

Measurement of Hbt. salinarum respiratory activity. The respiratory oxygen consumption was measured in Hbt. salinarum, an aerobic heterotroph. Archaea were exposed to various cultivated conditions as described in material and method (H. salinarum cells: cultivation and growth conditions). For each treatment, the rate of cellular respiratory activity was assayed in the same cell-suspension cultures before (salt shock) and after reactivation and in their corresponding supernatants. Air were bubble in $5 \mathrm{ml}$ of a suspension of archaea until dissolved oxygen was in near equilibrium with the atmosphere, then cells were placed into a thermostated cell at $37^{\circ} \mathrm{C}$ for respiration measurement. Oxygen consumption was quantified by using the Unisense oxygen microsensor. The respiratory data were normalized to $10^{8}$ cells. All experiments were done in triplicate.

Microscopy. Time lapse microscopy was performed at the cell-imaging (M4D) platform of the IBS/ISBG on a temperature and illumination controlled IX81 inverted microscope using 100x (1.49NA) oil immersion objective and Dichroic Interference Contrast (DIC) imaging through diascopic LED light illumination (CoolLED ${ }^{\mathrm{TM}}$ ). Images were collected by a sCMOS Zyla camera (Andor), every 30 minutes as Z-stacks (PRIOR Piezo stage, one image every $0.5 \mu \mathrm{m} ; 5 \mu \mathrm{m}$ range). Acquisitions were performed continuously for up to 96 hours and time/Z series were analyzed with ImageJ to select the best focal plane, and crop the region of interest from the acquired field of view, and further analyzed using Volocity (Quorum Technologies).

Sample preparation for the neutron scattering experiments. Between $500 \mathrm{ml}$ to $1.2 \mathrm{~L}$ of cultures were centrifuged at $4000 \times \mathrm{g}$ for $20 \mathrm{~min}$. For the salt shock and recovery experiments, cell pastes were gently suspended in culture medium containing $2.5,1.5$ and $0.5 \mathrm{M} \mathrm{NaCl}$ and incubated for one hour or 24 hours at $37^{\circ} \mathrm{C}$ with shaking. Culture of stressed cells were centrifuged at $6000 \times \mathrm{g}$ for $30 \mathrm{~min}$. The culture was then divided in two for NS experiments: (a) a pellet used for the shocked cells and (b) a cell paste gently re-suspended in $4.2 \mathrm{M} \mathrm{NaCl}$, incubated at $37^{\circ} \mathrm{C}$ with shaking for one hour and centrifuged at $6000 \mathrm{~g}$ for $30 \mathrm{~min}$, for the recovered cells. The shocked, recovered and control $(4.2 \mathrm{M} \mathrm{NaCl})$ pellets $(400 \mathrm{mg}$ each) were inserted in a $0.3-\mathrm{mm}$ path length gold-coated aluminum flat sample older. Elastic neutron scattering (EINS) spectra were collected at the high resolution back scattering spectrometer $\mathrm{IN} 13^{38}$ at the Institut Laue-Langevin (ILL), Grenoble, France. With an energy resolution of $8 \mu \mathrm{eV}$ (full width at half maximum, FWHM) and an accessible momentum transfer range of $0.2<\mathrm{Q}<4.9 \AA^{-1}$, IN13 allows the investigation of molecular motions on a time scale up to $100 \mathrm{ps}$ and with an amplitude from $1.3 \AA$ to $\sim 31 \AA^{37}$. Data were acquired in the temperature range $278-315 \mathrm{~K}$, above the freezing point of the water in order to avoid coherent scattering contributions arising from ice Bragg reflections. In order to optimize the signal to noise ratio, 4 hours of acquisition time per point were necessary. The program LAMP ${ }^{39}$ was used for data reduction, consisting in subtraction of the empty cell contribution and normalization with respect to a vanadium scan (a totally incoherent sample) to compensate for differences in detector efficiency and geometry. In order to avoid corrections from multiple scattering events, cell thickness and geometry were properly chosen to minimize neutron absorption from the sample (sample transmission $\sim 90 \%$ ).

\section{Data availability}

All data needed to evaluate the conclusions in the paper are present in the paper and/or the Supplementary Materials. Data digital object identifier https://doi.org/10.5291/ILL-DATA.8-04-817.

Received: 31 July 2019; Accepted: 4 December 2019;

Published online: 24 February 2020

\section{References}

1. Ionescu, D. et al. Microbial and chemical characterization of underwater fresh water springs in the dead sea. PLoS One 7(6), e38319 (2012).

2. Oren, A. Halophilic microbial communities and their environments. COBIOT 33, 119-24 (2015).

3. Ginzburg, M. \& Ginzburg, B. Z. Regulation of cell volume and ion concentrations in a. Halobacterium. J. Membr. Biol. 26, 153-71 (1976).

4. Gunde-Cimerman, N., Plemenitaš, A. \& Oren, A. Strategies of adaptation of microorganisms of the three domains of life to high salt concentrations. FEMS Microbiol. Rev 42, 353-75 (2018).

5. Madern, D., Ebel, C. \& Zaccai, G. Halophilic adaptation of enzymes. Extremophiles 4, 91-8 (2000).

6. Lanyi, J. K. Salt-dependent properties of proteins from extremely halophilic bacteria. Bacteriol. Rev. 38, 272-90 (1974).

7. Müller-Santos, M. et al. First evidence for the salt-dependent folding and activity of an esterase from the halophilic archaea Haloarcula marismortui. Biochim. Biophys. Acta 1791, 719-29 (2009).

8. Irimia, A. et al. The Oligomeric states of Haloarcula marismortui malate dehydrogenase are modulated by solvent components as shown by crystallographic and biochemical studies. J. Mol. Biol. 326, 859-73 (2003).

9. Ebel, C. et al. Solvent interactions of halophilic malate dehydrogenase. Biochemistry 41, 13234-44 (2002).

10. Costenaro, L., Zaccai, G. \& Ebel, C. Link between protein-solvent and weak protein-protein interactions gives insight into halophilic adaptation. Biochemistry 41, 13245-52 (2002).

11. Tadeo, X. et al. Structural basis for the aminoacid composition of proteins from halophilic archea. PLoS Biol. 7(12), e1000257 (2009).

12. Zaccai, G. Molecular dynamics in cells: A neutron view. BBA General Subjects, https://doi.org/10.1016/j.bbagen.2019.129475 (2019).

13. Tehei, M., Madern, D., Pfister, C. \& Zaccai, G. Fast dynamics of halophilic malate dehydrogenase and BSA measured by neutron scattering under various solvent conditions influencing protein stability. Proc. Natl. Acad. Sci. USA 98, 14356-61 (2001).

14. Marty, V. et al. Neutron scattering: a tool to detect in vivo thermal stress effects at the molecular dynamics level in micro-organisms. J. R. Soc. Interface 10, 20130003 (2013).

15. Vauclare, P. et al. Molecular adaptation and salt stress response of Halobacterium salinarum cells revealed by neutron spectroscopy. Extremophiles 19, 1099-107 (2015).

16. Zaccai, G. et al. Neutrons describe ectoine effects on water H-bonding and hydration around a soluble protein and a cell membrane. Sci. Rep. 6, 31434 (2016).

17. Franzetti, B. et al. Characterization of a novel complex from halophilic archaebacteria, which displays chaperone-like activities in vitro. J. Biol. Chem. 276, 29906-14 (2001).

18. Chamieh, H., Marty, V., Guetta, D., Perollier, A. \& Franzetti, B. Stress regulation of the PAN-proteasome system in the extreme halophilic archaeon Halobacterium. Extremophiles 16, 215-25 (2012). 
19. Leuko, S., Raftery, M. J., Burns, B. P., Walter, M. R. \& Neilan, B. A. Global protein-level responses of Halobacterium salinarum NRC1 to prolonged changes in external sodium chloride concentrations. J. Proteome Res. 8, 2218-25 (2009).

20. Becker, E. A. et al. Phylogenetically driven sequencing of extremely halophilic archaea reveals strategies for static and dynamic osmo-response. PLoS genetics 10, e1004784 (2014).

21. Coker, J. A., DasSarma, P., Kumar, J., Müller, J. A. \& DasSarma, S. Transcriptional profiling of the model archaeon Halobacterium sp. NRC-1: responses to changes in salinity and temperature. Saline Systems 3, 6 (2007)

22. Jensen, M. W. et al. Potassium stress growth characteristics and energetics in the haloarchaeon Haloarcula marismortui. Extremophiles 19, 315-25 (2015).

23. Dawson, K. S. et al. Quantitative fluorescence in situ hybridization analysis of microbial consortia from a biogenic gas field in Alaska’s Cook Inlet basin. Appl. Environ. Microbiol. 78, 3599-605 (2012).

24. Youssef, N. H. et al. Trehalose/2-sulfotrehalose biosynthesis and glycine-betaine uptake are widely spread mechanisms for osmoadaptation in the Halobacteriales. ISME J. 8, 636-49 (2014).

25. Oxley, A. P. et al. Halophilic archaea in the human intestinal mucosa. Environ. Microbiol. 12, 2398-410 (2010).

26. Purdy, K. J. et al. Isolation of haloarchaea that grow at low salinities. Environ. Microbiol. 6, 591-5 (2004).

27. Thombre, R. S., Shinde, V. D., Oke, R. S., Dhar, S. K. \& Shouche, Y. S. Biology and survival of extremely halophilic archaeon Haloarcula marismortui RR12 isolated from Mumbai salterns, India in response to salinity stress. Sci. Rep. 6, 25642 (2016).

28. Moissl-Eichinger, C. et al. Archaea are interactive components of complex microbiomes. Trends Microbiol. 26, $70-85$ (2018).

29. DasSarma, P. \& DasSarma, S. Survival of microbes in earth's stratosphere. Curr. Opin. Microbiol. 43, 24-30 (2018).

30. Zaccai, G. The ecology of protein dynamics. Curr. Phys. Chem. 3, 9-16 (2013).

31. Tehei, M. et al. Adaptation to extreme environments: macromolecular dynamics in bacteria compared in vivo by neutron scattering. EMBO Rep. 5, 66-70 (2004)

32. Colwell, R. R. Viable but nonculturable bacteria: a survival strategy. J. Infect. Chemother. 6, 121-5 (2000)

33. Joux, F. \& Lebaron, V. Use of fluorescent probes to assess physiological functions of bacteria at single-cell level. Microbes. Infect. 2, 1523-35 (2000).

34. Natali, F. et al. Changes in protein dynamics induced under Gdn-HCl denaturation. Appl. Phys. A 75, 1-3 (2002).

35. Franzetti, B. et al. Tetrahedral aminopeptidase: a novel large protease complex from archaea. EMBO J. 21(9), 2132-2138 (2002).

36. Fisher, R. A., Gollan, B. \& Helaine, S. Persistent bacterial infections and persister cells. Nat. Rev. Microbiol. 15, 453-464 (2017).

37. Megaw, J. \& Gilmore, B. F. Archaeal persisters: persister cell formation as a stress response in Haloferax volcanii. Front. Microbiol. 8, $1-10$ (2017).

38. Natali, F. et al. IN13 backscattering spectrometer at ILL: looking for motions in biological macromolecules and organisms. Neutron News 19, 14-18 (2008)

39. Richard, D., Ferrand, M. \& Kearley, G. Analysis and visualisation of neutron-scattering data. JNR 4, 33-39 (1996).

\section{Acknowledgements}

We acknowledge ILL for beam time. This work was supported by the French National Research Agency in the framework of the Investissements d'Avenir program (ANR-15-IDEX-02). The authors acknowledge the platforms of the Grenoble Instruct-ERIC center (ISBG; UMS 3518 CNRS-CEA-UJF-EMBL) within the Grenoble Partnership for Structural Biology (PSB). Platform access was supported by FRISBI (ANR-10-INBS-05-02) and GRAL, a project of the University Grenoble Alpes graduate school (Ecoles Universitaires de Recherche) CBHEUR-GS (ANR-17-EURE-0003). IBS acknowledges integration into the Interdisciplinary Research Institute of Grenoble (IRIG, CEA). We are very grateful to I. Fourquaux of the Center for Electron Microscopy Applied to the Biology (CMEAB) of Toulouse and to D. Fenel and B. Gallet of the IBS electronic microcopy platform in Grenoble for the SEM and TEM experiments, respectively. We are also deeply grateful for the supply of Unisense oxygen microsensor by M. Byrdin (IBS dynamop group in Grenoble).

\section{Author contributions}

All proposed and contributed to carry out, analyze and interpret experiments in their respective expertise (P.V., J.-P.K. and B.F. biochemistry and cell biology - F.N. and G.Z. neutron scattering) and participated in writing the manuscript. P.V. and F.N. designed and conducted experiments and wrote the paper. J.P. Kleman conducted experiments. B.F. and G.Z. supervised work and wrote the paper.

\section{Competing interests}

The authors declare no competing interests.

\section{Additional information}

Supplementary information is available for this paper at https://doi.org/10.1038/s41598-020-59681-1.

Correspondence and requests for materials should be addressed to B.F.

Reprints and permissions information is available at www.nature.com/reprints.

Publisher's note Springer Nature remains neutral with regard to jurisdictional claims in published maps and institutional affiliations.

Open Access This article is licensed under a Creative Commons Attribution 4.0 International License, which permits use, sharing, adaptation, distribution and reproduction in any medium or format, as long as you give appropriate credit to the original author(s) and the source, provide a link to the Creative Commons license, and indicate if changes were made. The images or other third party material in this article are included in the article's Creative Commons license, unless indicated otherwise in a credit line to the material. If material is not included in the article's Creative Commons license and your intended use is not permitted by statutory regulation or exceeds the permitted use, you will need to obtain permission directly from the copyright holder. To view a copy of this license, visit http://creativecommons.org/licenses/by/4.0/.

(C) The Author(s) 2020 